\title{
Sonication of Intramedullary Nails: Clinically-Related Infection and Contamination
}

\author{
J. Esteban ${ }^{1,3}$, E. Sandoval2,3, J. Cordero-Ampuero ${ }^{4}$, D. Molina-Manso ${ }^{1}$, A. Ortiz-Pérez ${ }^{1}$, \\ R. Fernández-Roblas ${ }^{1,3}$ and E. Gómez-Barrena ${ }^{*, 5}$
}

Departments of ${ }^{1}$ Clinical Microbiology and ${ }^{2}$ Orthopaedics, ${ }^{3}$ Bone and Joint Infection Unit, IIS-Fundación Jiménez Díaz, Madrid, Spain; ${ }^{4}$ Department of Orthopaedics, Hospital de la Princesa, Madrid, Spain; ${ }^{5}$ Department of Orthopaedics, IdiPaz-Hospital La Paz Institute for Health Research, Madrid, Spain

\begin{abstract}
Background and Aim: Sonication is currently considered the best procedure for microbiological diagnosis of implant-related osteoarticular infection, but studies in nail-related infections are lacking. The study aim was to evaluate implant sonication after intramedullary nail explantation, and relate it to microbiological cultures and clinical outcome.

Patients and Methods: A study was performed in two University Hospitals from the same city. Thirty-one patients with implanted nails were prospectively included, whether with clinical infection ( 8 cases) or without ( 23 cases). Retrieved nails underwent sonication according a previously published protocol. The clinical and microbiological outcome patient was related to the presence of microorganisms in the retrieved implant.

Results: Positive results appeared in $15 / 31$ patients ( 9 with polymicrobial infections) almost doubling those clinically infected cases. The most commonly isolated organisms were Staphylococcus epidermidis (19.2\%) and Staphylococcus aureus $(15.4 \%)$. A significant relationship was found between the presence of positive cultures and previous local superficial infection $(\mathrm{p}=0.019)$. The presence of usual pathogens was significantly related to clinical infection $(\mathrm{p}=0.005)$ or local superficial infection $(\mathrm{p}=0.032)$. All patients with positive cultures showed pain diminution or absence of pain after nail removal (15/15), but this only occurred in 8 (out of 16) patients with negative cultures.

Conclusions: In patients with previously diagnosed infection or local superficial infection, study of the hardware is mandatory. In cases where pain or patient discomfort is observed, nail sonication can help diagnose the implant colonization with potential pathogens that might require specific treatment to improve the final outcome.
\end{abstract}

Keywords: Intramedullary nail, orthopaedic infection, sonication, infection diagnosis, nail removal, implant-related infection.

\section{INTRODUCTION}

Infection is one of the most important complications in the treatment of fractures, leading to non-unions or established osteomyelitis. The incidence of infection related to osteosynthesis broadly varies according to the literature, from superficial surgical site infections to deep, severe problems, being more frequent in open fractures [1-3]. The evolution of these infections is a matter of major concern, as they can lead to severe impairment including amputation or even death of the patient due to sepsis.

Diagnosis of such an infection is crucial, because a proper identification of the responsible microorganism guides patient treatment with the best available antibiotic or combination of antibiotics [4]. Failure to identify this agent leads to an empiric therapy that can be inadequate for an specific pathogen. Although diagnostic protocols for wound infection are well known [5], diagnosis of infections associated with osteosynthesis material is controversial, and no clear protocol has been universally recommended, especially for intramedullary nails $[4,6,7]$. Many patients

*Address correspondence to this author at the Servicio de COT-A-1 ${ }^{\text {a }}$ Planta Hospital de Traumatología, Hospital La Paz, Po Castellana 261, Madrid 28046, Spain; Tel: +34917277085;

E-mails: egomezb@salud.madrid.org,enrique.gomezbarrena@uam.es with intramedullary nails request and undergo hardware removal due to ill-defined pain, consolidation problems or even purulent drainage, but infection is difficult to confirm microbiologically. In this sense, it is important to consider the biofilm development in the pathogenesis of infection [8]. Bacteria inside the biofilm can be difficult to discover if cultures of the surrounding tissues are the only diagnostic technique. Several procedures have been used to increase diagnostic effectivity, from biomaterial scraping or swabbing to culturing the whole implant in broth media $[6,7,9]$. However, all these procedures are less useful than sonication that has been recently established as the best diagnostic procedure for prosthetic joint-related infections $[10,11]$. Data also suggest that this technique could be useful in the diagnosis of osteosynthesis-related infections [10]. However, there are no clear data on the use of sonication in the diagnosis of nail-associated infection. The aim of this study is to evaluate implant-related infections through explanted nail sonication, considering microbiological culture results and clinical outcome.

\section{PATIENTS AND METHODS}

\section{Patients and Samples}

Thirty one patients were included in the study after referral for hardware removal. One intramedullary nail was 
removed per patient, and these nails had been previously implanted in the treatment of dyaphyseal fractures in the femur, tibia or humerus. Hardware removal was decided because of infection, pain or patient discomfort, and the patient was referred based on his or her request of having the nail removed.

Demographics, clinical symptoms and signs at the moment of revision surgery, antibiotic therapy, and final outcome were registered according to a previously developed protocol specifically including the presence and location of pain, the presence of local infection, the existence of non union, fracture features and reasons for surgery. All these were registered as individual variables.

During surgery, nails were aseptically removed and introduced in large sterile plastic bags. Three bags, one inside the other, were used by the scrub nurse to prepare the specimen. These were then sent to the Microbiology laboratory of one of the hospitals to be processed. If immediate processing was not available, samples were stored at $4^{\circ} \mathrm{C}$ during a maximum of 24 hours until processing. Tissue samples and wound exudates were taken according to the hospitals' protocols. Cases were included when clinical data were available and nail sonication was performed following the protocol, without contamination or bag damage.

\section{Sample Processing}

At the reference laboratory, samples were aseptically removed from the original bags and located into new, sterile plastic bags. Fifty mililiters of buffer phosphate were added, and bags were then closed. Samples were then sonicated according to a previously described protocol [10]. To avoid potential contaminations, several aspects were taken into consideration: Use of sterile plastic bags different from the original ones, careful inspection of the bags before and after sonication looking for leaks, use of changed distilled water for each sonication procedure, discharge of the water used in each sonication, and routine control cultures of the water used for sonication (all of them being negative). Moreover, uncommon nonfermenting gramnegative organisms in low counts were considered as contaminants and the result of these cultures was reported as negative. Conventional samples were processed according to conventional techniques, and culture media and incubation times were also those previously reported [10]. Cultures were quantified and the amount of bacteria was expressed in $\mathrm{CFU} / \mathrm{ml}$ for the $5 \mathrm{ml}$ of centrifuged sonicate. Isolated organisms were identified according to commonly reported protocols. We defined mixed anaerobic microbiota as the presence of more than 2 different species of anaerobic bacteria. Usual pathogens were defined as those organisms that are common cause of infections in both healthy and unhealthy patients. Antimicrobial susceptibility testing was performed using a disc-plate assay according to CLSI standards.

\section{Management of the Patients}

Patients with positive isolates were treated with antibiotics according to antimicrobial susceptibility and clinical criteria. If infection with purulent drainage was obtained, wide surgical debridement was performed, followed by antibiotic treatment. If non-union was present, surgical debridement was followed by external or internal fixation as required. Postoperative antibiotic therapy was chosen according to the individual susceptibility pattern of each pathogen.

\section{Statistical Analysis}

We performed the analysis considering a null hypothesis of no difference between clinical diagnosis of infection (based on clinical signs and conventional culture) and culture from the nail sonication solution in the bag, especially regarding the outcome of the patients.

Comparison of qualitative variables was performed using Fisher's exact test and Chi-square test. For quantitative variables, Bartlett test was used to check the normality of the distribution. ANOVA or Kruskal-Wallis tests were subsequently used to compare the data. To perform statistical analysis of colony counts, all samples with $\geq 100,000$ $\mathrm{CFU} / \mathrm{ml}$ were grouped as $100,000 \mathrm{CFU} / \mathrm{ml}$. All statistical calculations were performed with SPSS 17.0 software (SPSS Inc., Chicago IL, USA).

\section{RESULTS}

Thirty-one patients were included in this study, with a mean \pm SD age of $50.35 \pm 19.35$ years. No significant differences were found between infected and non-infected patients $(55.5 \pm 25.34$ and $48.56 \pm 17.13$ years respectively). Sixteen patients were male $(51.6 \%)$ and 15 were female $(48.4 \%)$. Again, no gender differences were found related to the presence of clinical infection. Male patients were younger $(41.01 \pm 11.78)$ than female patients $(60.26 \pm 21.21)$, a statistically significant difference $(p=0.0067$, KruskalWallis test). Characteristics of the cases are shown in Table 1.

Clinical infection was diagnosed any time after the fracture in 8 different patients ( 3 males and 5 females), and defined as visible purulence surrounding the nail surgical entry point and/or the fracture site, in case of open fracture. Among all cases (infected and non-infected), 8 patients had open fractures ( 2 of them were infected); non union was diagnosed in 10 cases, and local superficial infection in 10 cases. Pain was detected in 25 cases; in 13 out of these 25 , it was located at the previous fracture site. The main reasons for nail removal was pain in 14 cases, followed by the presence of a persistent infection (6 cases), and an established nonunion (4 cases). Fifteen patients received documented treatment with antibiotics previous to nail retrieval surgery, 7 of them received only short courses of antibiotics related to mild infection of other origin.

Fifteen cases had positive results of cultures from the sonicated samples (Table 2). Only 1 case of clinical infection had negative cultures (this case had a positive conventional culture for Enterococcus spp.), while 8 cases without a previous diagnosis of infection showed positive results. In 9 of the culture-positive cases, more than one species were isolated (polymicrobial infections). The isolated species were Staphylococcus epidermidis (5 strains, $19.2 \%$ ), Staphylococcus aureus (4 strains, $15.4 \%$ ), mixed anaerobic microbiota (3 cases, $11.5 \%$ ), Propionibacterium acnes (2 strains, $7.7 \%$ ), Peptostreptococcus sp. (2 strains, $7.7 \%$ ) and one strain each of Enterococcus faecalis, Providencia stuartii, Streptococcus milleri, Staphylococcus warnerii, 
Table 1. Characteristics of the Patients

\begin{tabular}{|c|c|c|c|c|c|c|c|c|c|}
\hline Patient \# & Age & Sex & $\begin{array}{l}\text { Clinical Diagnosis } \\
\text { of Infection }\end{array}$ & $\begin{array}{c}\text { Open } \\
\text { Fracture }\end{array}$ & $\begin{array}{c}\text { Local Superficial } \\
\text { Infection }\end{array}$ & $\begin{array}{l}\text { Non } \\
\text { Union }\end{array}$ & $\begin{array}{c}\text { Previous Antibiotic } \\
\text { Therapy }\end{array}$ & $\begin{array}{c}\text { Pain } \\
\text { Location }\end{array}$ & $\begin{array}{c}\text { Cause of } \\
\text { Nail Removal }\end{array}$ \\
\hline 1 & 42 & $\mathrm{~F}$ & No & No & No & Yes & No & Other zone & Non union surgery \\
\hline 2 & 40 & M & No & Yes & No & No & Yes & Fracture focus & Other \\
\hline 3 & 41 & M & No & No & Yes & Yes & Yes & Other zone & Pain \\
\hline 4 & 75 & $\mathrm{~F}$ & Yes & No & Yes & Yes & Yes & Other zone & Infection \\
\hline 5 & 78 & $\mathrm{~F}$ & No & No & No & No & No & Fracture focus & Pain \\
\hline 6 & 59 & M & No & No & Yes & Yes & Yes & Other zone & Pain \\
\hline 7 & 40 & $\mathrm{~F}$ & No & No & Yes & No & No & Fracture focus & Pain \\
\hline 8 & 85 & $\mathrm{~F}$ & No & No & No & Yes & No & Fracture focus & Non union surgery \\
\hline 9 & 58 & $\mathrm{~F}$ & No & No & No & No & No & Other zone & Pain \\
\hline 10 & 29 & $\mathrm{~F}$ & Yes & No & Yes & No & Yes & Fracture focus & Infection \\
\hline 11 & 49 & $\mathrm{~F}$ & No & No & No & No & No & Other zone & Pain \\
\hline 12 & 35 & M & Yes & Yes & Yes & Yes & Yes & Fracture focus & Non union surgery \\
\hline 13 & 27 & M & No & No & No & No & No & No & Other \\
\hline 14 & 64 & M & No & No & No & No & No & Other zone & Pain \\
\hline 15 & 89 & $\mathrm{~F}$ & Yes & No & Yes & No & No & Fracture focus & Infection \\
\hline 16 & 36 & M & No & No & No & No & No & No & Other \\
\hline 17 & 43 & M & Yes & Yes & Yes & Yes & Yes & Fracture focus & Infection \\
\hline 18 & 16 & $\mathrm{~F}$ & No & No & No & No & Yes & Fracture focus & Pain \\
\hline 19 & 53 & M & No & Yes & No & Yes & Yes & Fracture focus & Pain \\
\hline 20 & 47 & M & No & No & No & No & Yes & No & Other \\
\hline 21 & 24 & M & Yes & No & Yes & No & Yes & Fracture focus & Infection \\
\hline 22 & 53 & $\mathrm{~F}$ & No & Yes & No & No & Yes & Other zone & Other \\
\hline 23 & 38 & M & No & No & No & No & No & Other zone & Pain \\
\hline 24 & 52 & M & No & Yes & No & Yes & Yes & Fracture focus & Non union surgery \\
\hline 25 & 47 & $\mathrm{~F}$ & No & Yes & No & No & No & Other zone & Pain \\
\hline 26 & 75 & $\mathrm{~F}$ & No & No & No & No & No & No & Other \\
\hline 27 & 75 & $\mathrm{~F}$ & Yes & No & No & No & No & Other zone & Pain \\
\hline 28 & 61 & $\mathrm{~F}$ & No & Yes & No & Yes & Yes & Fracture focus & Pain \\
\hline 29 & 32 & M & No & No & No & No & No & No & Other \\
\hline 30 & 24 & M & No & No & No & No & No & Other zone & Pain \\
\hline 31 & 74 & $\mathrm{~F}$ & Yes & No & Yes & No & Yes & No & Infection \\
\hline
\end{tabular}

Mycobacterium fortuitum, Pseudomonas stutzerii, Ralstonia pickettii, Sphingomonas paucimobilis, Micrococcus sp. and Candida parapsilosis. Colony count of patients with clinical infection averaged $47,120 \mathrm{CFU} / \mathrm{ml}( \pm 44,726$, range 200 to $100,000 \mathrm{CFU} / \mathrm{ml})$, and $27,840 \mathrm{CFU} / \mathrm{ml}( \pm 39,533$, range 200 to $100,000 \mathrm{CFU} / \mathrm{ml}$ ) for patients without clinical infection. This difference was not statistically significant $(\mathrm{p}=0.15$, ANOVA test). Moreover, colony counts were not affected from previous antimicrobial therapy $(\mathrm{p}=0.86$, ANOVA test). Conventional cultures were performed in 24 patients, being positive in 6 cases (all of them, clinically infected patients). Correlation between positive results appeared in 4 cases (1 S. aureus, 1 P. acnes, $1 S$. milleri + anaerobic bacteria, and 1 P. stuartii + anaerobic bacteria). One case showed different results between both types of samples $(P$. aeruginosa in wound exudates, and Enterococcus faecalis + $P$. prevotii in sonicated samples), and one case had positive result in wound exudates (Enterococcus sp.) but negative results in sonicate samples.

No statistical association was detected between the presence of positive cultures and presence of pain $(p=0.35)$, location of pain $(\mathrm{p}=0.12)$, non union $(\mathrm{p}=0.6)$, open fracture $(p=0.12)$ or previous antibiotic therapy $(p=0.11)$, but there was a relationship between the presence of local superficial infection and positive cultures $(\mathrm{p}=0.019$, Fisher's exact test). 
Table 2. Characteristics of the Microorganisms

\begin{tabular}{|c|c|c|c|c|}
\hline Patient \# & $\begin{array}{c}\text { Total Colony Count }(\mathrm{CFU} / \mathrm{ml}) \text { in } \\
\text { Sonication Cultures }\end{array}$ & Microbial Isolates & Antibiotic Therapy & Outcome \\
\hline 1 & 1200 & S. aureus S. epidermidis (2 different strains) & Yes & Improvement \\
\hline 2 & & & No & Improvement \\
\hline 3 & 50000 & S. paucimobilis & No & Improvement \\
\hline 4 & 60000 & S. aureus, S. epidermidis, $S$. warneri & Yes & Improvement \\
\hline 5 & 1000 & M. fortuitum, Candida parapsilosis & & Improvement \\
\hline 6 & & & Yes & Improvement \\
\hline 7 & 100000 & P. acnes, $P$. stutzeri & No & Improvement \\
\hline 8 & & & No & NA \\
\hline 9 & & & & NA \\
\hline 10 & 200 & S. aureus & Yes & NA \\
\hline 11 & & & No & Improvement \\
\hline 12 & 100000 & E. faecalis, $P$. prevotii & Yes & Improvement \\
\hline 13 & & & Yes & NA \\
\hline 14 & 50000 & S. epidermidis & Yes & Improvement \\
\hline 15 & 100000 & P. stuartii, mixed anaerobic microbiota & Yes & Improvement \\
\hline 16 & & & No & Improvement \\
\hline 17 & & & Yes & Persistence of infection \\
\hline 18 & 1000 & R. pickettii & No & Improvement \\
\hline 19 & & & No & Improvement \\
\hline 20 & 75000 & S. epidermidis, Micrococcus spp. & Yes & Improvement \\
\hline 21 & 100000 & Mixed anaerobic microbiota & Yes & NA \\
\hline 22 & & & No & Improvement \\
\hline 23 & & & No & Improvement \\
\hline 24 & & & Yes & Persistence of pain \\
\hline 25 & & & No & Persistence of pain \\
\hline 26 & & & No & Improvement \\
\hline 27 & 11000 & Peptostreptococcus spp., P. acnes & Yes & Improvement \\
\hline 28 & 200 & S. aureus & Yes & Improvement \\
\hline 29 & & & No & NA \\
\hline 30 & & & No & Persistence of pain \\
\hline 31 & 100000 & S. milleri, Mixed anaerobic microbiota & Yes & NA \\
\hline
\end{tabular}

NA: Data not available.

An association was observed between infection and positive cultures with usual pathogens $(p=0.005$, Fisher's exact test). Paired analysis also showed a relationship between the presence of usual pathogens and the presence of local superficial infection $(\mathrm{p}=0.032)$, but no other relationship was found for the rest of the other variables.

Patients with clinical diagnosis of infection and those without such diagnosis but with a positive culture for usual pathogens, were treated with antibiotics following microorganism susceptibility tests. The final outcome of the hardware removal procedure was collected at minimum 6 months in 24 patients. One patient underwent limb amputation because of untreatable infection, and was excluded. Six patients were lost after surgery and were also excluded. Nine out of 24 patients had pain at 6 or more months, including 5 patients with negative culture $(41.7 \%)$ and 4 with positive culture (33.3\%), a non-significant difference. Eight patients with negative cultures $(66.7 \%)$ and all 12 patients with positive cultures (100\%) showed pain diminution after nail removal, a statistically significant difference $(p=0.046$, Fisher's exact test). Fifteen patients received antibiotic therapy after nail removal, including 4 with negative cultures (three with wound infections in another location and one with clinical diagnosis of infection 
despite negative cultures). Although no relationship could be found between antibiotic therapy and improvement of symptoms, all patients with usual pathogens were treated. Three patients with positive cultures which were not treated had isolates that were classified as unusual pathogens ( $1 P$. acnes, 1 S. paucimobilis and 1 R. pickettii).

\section{DISCUSSION}

Present interest on implant-related infection has been influenced by the development of the biofilm concept $[8,12$ 17]. According to this idea, bacteria are tightly attached to the implant surface and routine techniques would not detect them. Therefore, nail infection is usually diagnosed only after clinically relevant infection occurs, with drainage, bone changes, or other severe signs present in the case. Several laboratory techniques have been used to improve the diagnosis of infection in doubtful or uncertain cases $[4,18]$. Recently, different reports stated the usefulness of sonication for the diagnosis of prosthetic joint infections [10, 11, 15]. However, no broad studies have applied these protocols for the diagnosis of fracture fixation device-related infection. In our study, a previously described technique for devicerelated infection diagnosis was used [10]. We found 8 cases with positive cultures but without clinical disease, and only one case of infection gave negative results. When we analyzed the results of non-infected patients, we found 4 cases with positive cultures for environmental organisms $(S$. paucimobilis, R. pickettii, $P$. stutzeri and M. fortuitum). These organisms can be usually found in water-related systems. Except for the case of $S$. paucimobilis isolation (which gave 50,000 $\mathrm{CFU} / \mathrm{ml}$ ), all other environmental organisms gave low colony counts (less than $1,000 \mathrm{CFU} / \mathrm{ml}$ ), suggesting that they can be contaminants (no statistics have been performed because the low number of such organisms). Contamination due to leaks in the plastic bags used for sonication has been previously described [19], and this finding prompted to the use of rigid containers to avoid such contaminations $[11,18]$. Nevertheless, these studies have been performed in orthopaedic joint prosthesis, which have less length than nails. Nails used for osteosynthesis were 30 to $50 \mathrm{~cm}$ long, and no rigid containers could be used for sonication purposes; therefore plastic bags were used, controlling the bags for leakage and changing the distilled water of the sonicator after every case. Other sources of potential contamination could be related to the nail manufacture. This is improbable, as sterilization protocols on surgical devices are standardized in the different orthopaedic companies to comply with good manufacturing practices. The retrieved nails were obtained from five major manufacturers, and no association with positive cultures was detected in any of them. Moreover, sterilization of large batches of implants may not be homogenous, and hollow nails might allocate low number of environmental microorganisms that are so infrequent in the Hospital cultures. In our series, hollow nail versus solid nail contamination could not be clarified and warrants further work. In our opinion, the isolation of potentially contaminant organisms from long nail samples must be carefully evaluated, although they can be easily differentiated from true infections. Furthermore, the clinical outcome of patients with these potentially contaminated implants was benign, and at a minimum 6 months follow-up, all these patients did not present related symptoms. The source of these potential contaminations and the potential development of low grade infections from these microorganisms are uncertain. The sonication protocol seems to offer safety regarding contamination, if we compare our findings in nails with previous findings in joint prosthesis and plates [10].

Other non-environmental organisms have been isolated from apparently not infected samples, including $2 S$. aureus, $2 S$. epidermidis, and $1 P$. acnes. Interestingly, both $S$. epidermidis and the $P$. acnes isolates recorded high colony counts $(50,000 \mathrm{CFU} / \mathrm{ml}$ for $S$. epidermidis and $>100,000$ $\mathrm{CFU} / \mathrm{ml}$ for $P$. acnes), the $P$. acnes isolate recording the highest colony counts of all the samples. Moreover, both $S$. aureus isolates showed low colony counts (500 and 200 $\mathrm{CFU} / \mathrm{ml}$ ). There are reports in the literature that detected also similar bacteria in non-infected cases. One of them considered them in possible contaminants [6], while other reports suggest that they are probably subclinical infections $[9,20]$. We agree with these later reports, and we suggest that they might have not been clinically evident yet due to a low pathogenic potential of the organisms or a low number of bacteria present in the sample of an early retrieved implant.

Another interesting issue is the lack of efficacy in previous antibiotic therapy. Because sessile bacteria are phenotypically resistant to antibiotics [8,21], this could explain the isolation of apparently susceptible organisms from samples obtained after antibiotic therapy.

Based on these low-grade potential infections, nail removal could be recommended in cases where pain or patient discomfort is observed, although some data suggest that a good outcome and fracture union (but not infection healing) could be obtained without hardware removal [22]. Despite no clear association in our series and from the etiopathogenic point of view, a low-grade or a subclinical infection cannot be ruled out unless the implant is studied, and revision surgery is advised. In fact, a difference between culture positive and culture negative patients can be found in the outcome (all patients with positive cultures improved after nail removal, while $1 / 3$ of culture-negative cases did not improve), which suggest that removal of a potentially infected nail is highly important for patient improvement, even with low-grade infections. The fact that all patients with usual pathogens were specifically treated, even in the absence of clear infection symptoms, could have a definite role in their final outcome. Furthermore, local infection can be considered an indication of nail removal, as we were able to confirm that local infection was significantly associated with implant colonization. Although implant colonization may not imply observable clinical infection, the possibility of microorganism extension within the medullar canal with the potential severity of this infection, also outline the need for revision surgery. In fact, this reasoning is the cause that we treated all patients with potentially significant isolates, despite the absence of clinical symptoms in some cases. However, the fact that some cases could be treated without implant removal [22] must be taken into account. Fracture union and adequate infection treatment must be considered together before the final decision is taken about the optimal treatment of one patient. 
In conclusion, the removal and microbiological study of the hardware in patients with previous diagnosis of infection is mandatory, based on our findings. On the other hand, in cases where pain or patient discomfort are the guiding symptoms, nail sonication would help ruling out implant colonization with potential pathogens that might require specific treatment to improve the final outcome.

\section{ACKNOWLEDGEMENTS}

Part of this work was funded by grants from the Comunidad de Madrid (S2009/MAT-1472) and from the CONSOLIDER-INGENIO Program (FUNCOAT-CSD200800023). DMM was funded by a grant from the Fundación Conchita Rábago de Jiménez Díaz.

\section{CONFLICT OF INTEREST}

No conflict of interest exists for any of the authors.

\section{REFERENCES}

[1] Court-Brown CM, Keating JF, McQueen MM. Infection after intramedullary nailing of the tibia. J Bone Joint Surg Br 1992; 74B: $770-4$.

[2] Darouiche RO. Treatment of infections associated with surgical implants. New Engl J Med 2004; 350: 1422-9.

[3] Harwood PJ, Giannoudis PV, Probst C, Krettek C, Pape HC. The risk of local infective complications after damage control procedures for femoral shaft fracture. J Orthop Trauma 2006; 20(3): 181-9.

[4] Trampuz A, Zimmerli W. Diagnosis and treatment of infections associated with fracture-fixation devices. Injury 2006; 37: S59-66.

[5] Trampuz A, Widmer AF. Infections associated with orthopedic implants. Curr Opin Infect Dis 2006; 19: 349-56.

[6] Moussa FW, Anglen JO, Gehrke JC, Christensen G, Simpson WA. The significance of positive cultures from orthopaedic fixation devices in the absence of clinical infection. Am J Orthop 1997; 26(9): 617-20

[7] Singer PJ, Seligson D. What's in a nail? J Orthop Trauma 1990; 4(3): 331-5.

[8] Costerton JW. Biofilm theory can guide the treatment of devicerelated orthopaedic infections. Clin Orthop Relat Res 2005; 437: 711.
[9] Dobbins JJ, Seligson D, Raff MJ. Bacterial colonization of orthopedic fixation devices in the absence of clinical infection. J Infect Dis 1988; 158(1): 203-5.

[10] Esteban J, Gomez-Barrena E, Cordero J, Martin-de-Hijas NZ, Kinnari TJ, Fernandez-Roblas R. Evaluation of quantitative analysis of cultures from sonicated retrieved orthopedic implants in diagnosis of orthopedic infection. J Clin Microbiol 2008; 46(2): 488-92.

[11] Trampuz A, Piper KE, Jacobson MJ, et al. Sonication of removed hip and knee prostheses for diagnosis of infection. New Engl J Med 2007; 357: 654-63.

[12] Hall-Stoodley L, Costerton JW, Stoodley P. Bacterial biofilms: from the natural environment to infectious diseases. Nat Rev Microbiol 2004; 2: 95-108.

[13] Hall-Stoodley L, Stoodley P. Biofilm formation and dispersal and the transmission of human pathogens. Trends Microbiol 2005; 13(1): 7-10.

[14] Schmidt AH, Swiontkowski MF. Pathophysiology of infections after internal fixation of fractures. J Am Acad Orthop Surg 2000; 8: 285-91.

[15] Holinka J, Bauer L, Hirschl AM, Graninger W, Windhager R, Presterl E. Sonication cultures of explanted components as an addon test to routinely conducted microbiological diagnostics improve pathogen detection. J Orthop Res 2011; 29(4): 617-22.

[16] Piper KE, Jacobson MJ, Cofield RH, et al. Microbiologic diagnosis of prosthetic shoulder infection by use of implant sonication. J Clin Microbiol 2009; 47(6): 1878-84.

[17] Sampedro MF, Huddleston PM, Piper KE, et al. A biofilm approach to detect bacteria on removed spinal implants. Spine 2010; 35(12): 1218-24.

[18] Trampuz A, Zimmerli W. Diagnosis and treatment of implantassociated septic arthritis and osteomyelitis. Curr Infect Dis Rep 2008; 10: 394-403.

[19] Trampuz A, Piper KE, Hanssen AD, et al. Sonication of explanted prosthetic components in bags for diagnosis of prosthetic joint infection is associated with risk of contamination. J Clin Microbiol 2006; 44(2): 628-31.

[20] Marsh J, Nepola J, Seabold J. Subclinical infection in delayed/nonunion of fractures. Orthop Trans [Abstract] 1989; 13: 757.

[21] Donlan RM. Biofilm formation: a clinically relevant microbiological process. Clin Infect Dis 2001; 33: 1387-92.

[22] Berkes M, Obremskey WT, Scannell B, Ellington JK, Hymes RA, Bosse M. Maintenance of hardware after early postoperative infection following fracture internal fixation. J Bone Joint Surg Am 2010; 92(4): 823-8. 